\title{
La vidange d'un grand réservoir considérée comme une expérience de biomanipulation. Premiers résultats : contrôle exercé par les poissons sur la structure et la composition biochimique du plancton
}

\author{
P. Franciscol \\ J.N. Tourenq ${ }^{1}$
}

Mots clés : retenue, vidange, biomanipulation, zooplancton, relations trophiques, composition biochimique.

L'évolution de la structure du peuplement zooplanctonique du lac de Pareloup (Aveyron, France) ainsi que la composition biochimique du plancton, ont été suivies au cours de deux années : en 1993, lors de la vidange progressive de cette retenue et en 1994, durant la phase de recolonisation du milieu.

Les Crustacés planctoniques sont essentiellement représentés par Daphnia longispina, Ceriodaphnia quadrangula, Bosmina longirostris, Cyclops vicinus vicinus, Acanthocyclops robustus et Eudiaptomus vulgaris. Les Cladocères dominent le peuplement durant l'hiver et le début du printemps 1993. En 1994, leur développement est maximal en mai puis ils restent proportionnellement bien représentés en été. Les Cyclopides constituent la majeure partie de la communauté en juin 1993 et durant le début de la phase de recolonisation (automne 1993 - hiver 1994). La densité moyenne maximale des Crustacés (67 ind./litre) est observée en octobre 1993 juste après la remise en eau.

L'impact potentiel du zooplancton sur la communauté phytoplanctonique révèle le rôle prépondérant des petits brouteurs de janvier à juin 1993. En revanche, ce sont les grands brouteurs avec en moyenne $67 \%$ du volume potentiel d'eau filtrée, qui constituent d'octobre 1993 à juin 1994, les principaux régulateurs de la population algale. La biomasse moyenne au printemps 1993 est de $59 \mu \mathrm{g}$ PS/litre, celle enregistrée pour la même saison en 1994 est de $103 \mu \mathrm{g}$ PS/litre. Une augmentation significative des teneurs en lipides et protéines est notée dans le plancton en 1994.

La diminution du contrôle exercé par les poissons planctonophages éliminés lors de la vidange ( 80 tonnes) explique les variations de la structure et de la composition biochimique du peuplement planctonique.

The draining of a large reservoir considered as an experimental biomanipulation. First results : fish control on the structure and biochemical composition of plankton

Keywords : reservoir, draining, biomanipulation, zooplankton, trophic relationships, biochemical composition.

The structural evolution of zooplankton and the biochemical composition of plankton in Lake Pareloup (Department of Aveyron, France) was studied for a two year periods : during the progressive draining of this reservoir in 1993, and during the recolonisation phase in 1994.

The planktonic crustacean are essentially composed of Daphnia longispina, Ceriodaphnia quadrangula, Bosmina longirostris, Cyclops vicinus vicinus, Acanthocyclops robustus and Eudiaptomus vulgaris. The Cladocerans dominated the population during winter and in early spring 1993. In 1994, their development was maximal during May and they remained proportionally well represented in summer. The Cyclopoids constituted the main part of the community in june 1993 as well as at the beginning of the recolonisation phase (autumn winter 1993-1994). The maximum average crustacean density (67 ind./lit) was observed in October 1993 just after the water filling.

The potential impact of the zooplankton on the phytoplanktonic community reveals the preponderant role of small grazers from January to June 1993. However, large grazers with an average of $67 \%$ of the potential volume of filtered water represented the prime regulators of algal populations from October to June 1994 . The average biomass in spring 1993 was about $59 \mu \mathrm{g}$ DW/liter whilst the one recorded from the same season in 1994 was about $103 \mu \mathrm{g}$ DW/iter. A significant increase of lipid and protein content of plankton was noted in 1994.

The decrease of the control effected by planktophagous fish, eliminated during the draining of the reservoir ( 80 tons) explains the variations of structure and biochemical composition of the planktonic population.

1. Centre d'Écologie des Systèmes Aquatiques Continentaux, UMR C 5576CNRS, Université Paul Sabatier, 118 route de Narbonne, F-31062 Toulouse Cedex 04, France. 


\section{Introduction}

La gestion des écosystèmes lacustres, dans lesquels la communauté pisciaire revêt de plus en plus d'importance, ne peut être envisagée sans une bonne connaissance des relations trophiques qui existent dans ces milieux.

Selon la théorie de la «cascade trophique» proposée par Carpenter et al. (1985), les différents niveaux de la chaîne alimentaire sont étroitement liés et dépendent essentiellement de l'influence des facteurs de type descendant («top-down»). En revanche, McQueen et al. (1986, 1989, 1992), soulignent l'importance des effets de type ascendant («bottom-up»), en particulier dans la régulation des niveaux inférieurs du réseau trophique. Toutefois, les relations prédateurs-proies restent notables lorsqu'elles s'appliquent aux niveaux supérieurs. De plus, l'impact de chaque type d'effet régissant une composante donnée du réseau dépend du statut trophique du lac.

Concernant les milieux eutrophes, les modifications des communautés zooplanctoniques et phytoplanctoniques soumises à des changements du stock de prédateurs sont rapportées par plusieurs auteurs (Havens 1993, Carvalho 1994, Meijer et al. 1994).

Cependant il n'existe que peu de travaux explicitant à l'échelle d'un grand lac les conséquences d'une modification du réseau trophique en place sur les communautés planctoniques (Evans 1990, Rudstam et al. 1993).

La vidange réglementaire du lac-réservoir de Pareloup en juin 1993 a entraîné des modifications importantes du stock de poissons peuplant la retenue. Il a paru intéressant de suivre les variations saisonnières du peuplement microcrustacés pendant et après la vidange afin de vérifier le poids respectif des effets de type descendant et ascendant régissant l'évolution des zooplanctontes à l'échelle d'une telle retenue. Une précédente étude menée sur cet écosystème (Ferroni et al. 1996) a montré que les variations de la structure des populations zooplanctoniques et phytoplanctoniques ont une incidence sur la composition biochimique du plancton. Dans le but de compléter ces résultats, il a semblé opportun de suivre, lors de la recolonisation du milieu, l'évolution des teneurs en protéines et lipides du plancton nécessaire à la nouvelle communauté pisciaire de la retenue.

\section{Site, matériel et méthodes}

Le lac de Pareloup est située sur le plateau du Lévézou (Aveyron, France) à une altitude de 800 mètres. Qualifié de mésotrophe, ce plan d'eau d'une superficie de 1260 ha se place au cinquième rang des retenues hydroélectriques de France. Avec une profondeur maximale de 35 mètres et une profondeur moyenne de 12,5 mètres son volume atteint 168 millions de mètres cubes. Une stratification thermique se met en place de juin à septembre.

La vidange du lac de Pareloup a débuté en décembre 1992. Le volume de la retenue a été progressivement amené d'environ 168 millions de $\mathrm{m}^{3}$ à 0,3 millions de $\mathrm{m}^{3}$ en juin 1993, juste avant l'ouverture des vannes de fond. Après une période d'assec (juillet à septembre 1993), le bassin a atteint à nouveau son volume normal d'exploitation en février 1994. Les variations de niveau du plan d'eau sont représentées par la figure 1 pour les deux années d'étude.

La biomasse pisciaire de la retenue, principalement représentée par le gardon (Rutilus rutilus, L.), la brème (Abramis brama, L.) et la perche (Perca fluviatilis, L.), a été estimée avant la vidange à $68 \mathrm{~kg} \cdot \mathrm{ha}^{-1}$ à la cote maximale du lac (805 mètres) (Richeux et al. 1994a).

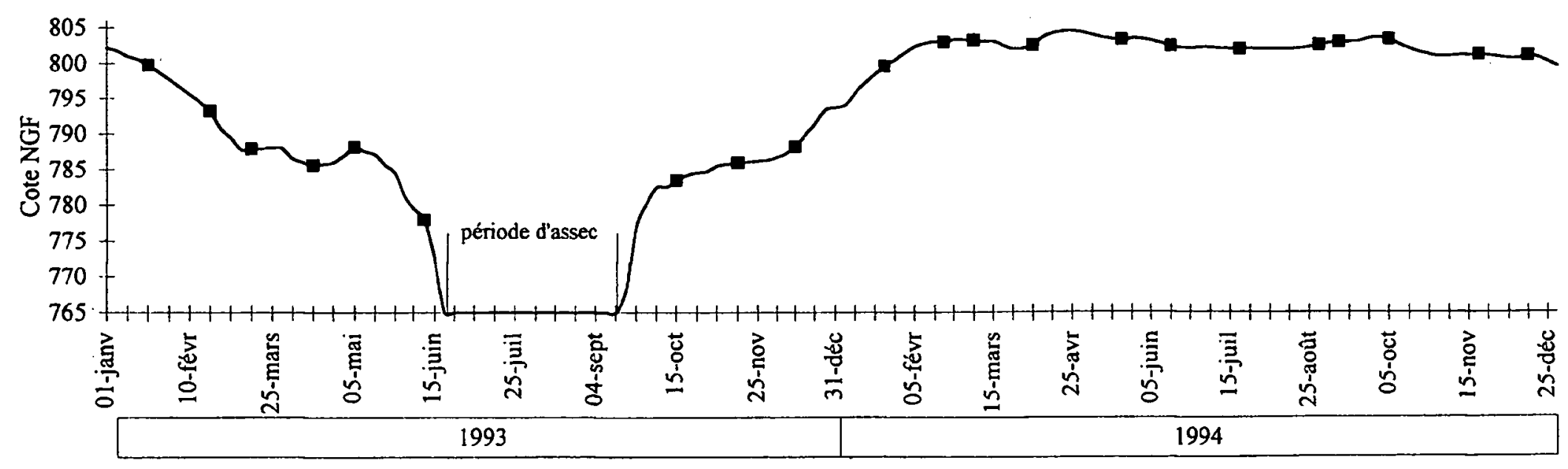

Fig. 1. Variations annuelles de la cote du lac (NGF). Les symboles (carrés noirs) indiquent les jours de prélèvement.

Fig. 1. Annual variations of the reservoir level (NGF). Symbols (blacks squares) indicates sampling days. 
La diminution du volume d'eau a entraîné une concentration des poissons de l'ordre de 150 à $200 \mathrm{~kg} \cdot \mathrm{ha}^{-1}$. Après la vidange, la structure du peuplement piscicole est caractérisée par une diminution considérable de sa biomasse et par une baisse de la proportion des planctonophages. La brème a totalement disparu et le gardon ne représente plus l'essentiel de la population. En 1994 , la biomasse estimée est inférieure à $20 \mathrm{~kg} \cdot \mathrm{ha}^{-1}$. Le peuplement est alors constitué en proportions presque identiques de piscivores (truites, brochets et sandres) et de planctonophages (gardons et perches), représentant respectivement 26 et $30 \%$ de la biomasse totale. Ce sont les espèces à tendance benthophages (tanches et carpes) qui dominent le peuplement en $1994(44 \%)$.

L'échantillonnage a été réalisé chaque mois durant deux périodes d'études :

- de janvier à juin 1993 (vidange progressive de la retenue),

- d'octobre 1993 (remise en eau) à décembre 1994.

Les données physico-chimiques correspondantes sont rapportées dans le tableau 1 . Les relevés sont effectués dans la zone pélagique à l'aide d'une bouteille de type Van Dorn ( 5 litres) aux profondeurs suivantes :
$0,5,2,5,5,10,15,20$ et 25 mètres. Pour chaque profondeur échantillonnée après homogénéisation du prélèvement, 1,5 litres sont immédiatement formolés ( $4 \%$ en solution finale). Les organismes sont dénombrés et mesurés à la loupe binoculaire $(\mathrm{G}: 12,5 \times 4)$.

Les densités sont exprimées en nombre d'individus par litre sur toute la colonne d'eau par la moyenne pondérée de l'effectif de chaque tranche d'eau prospectée.

Les valeurs de biomasse sont calculées à partir des relations taille-poids empruntées dans la littérature (Dumont et al. 1975, Bottrell et al. 1976).

Une classification fonctionnelle (Sprules 1984) basée sur la taille des organismes et leur écologie trophique permet d'appréhender les relations phytoplancton-zooplancton. Les Crustacés sont ainsi divisés en :

- petits brouteurs (Cladocères de taille inférieure à $0,5 \mathrm{~mm}$ et nauplii de Copépodes),

- grands brouteurs (Cladocères de taille supérieure à $0,5 \mathrm{~mm}$ ),

- Calanoïdes herbivores (copépodites et adultes de Diaptomides),

- carnivores (copépodites et adultes de Cyclopides). Cette dernière catégorie regroupe plutôt des Crustacés omnivores que des carnivores stricts.

Tableau 1. Données physico-chimiques relevées en 1993 et 1994 dans la retenue de Pareloup.

Table 1. Physicochemical data obtained in 1993 and 1994 in Pareloup reservoir.

\begin{tabular}{|c|c|c|c|c|c|c|c|c|c|c|c|c|c|c|}
\hline & & \multicolumn{2}{|c|}{ températures $\left({ }^{\circ} \mathrm{C}\right)$} & \multicolumn{2}{|c|}{ Oxygène $(\mathrm{mg} /)$} & \multicolumn{2}{|c|}{ Chlorophylle a. $(\mu \mathrm{g} / \mathrm{l})$} & \multicolumn{2}{|c|}{$\mathrm{SIO}_{2}(\mathrm{mg} / \mathrm{l})$} & \multicolumn{2}{|c|}{ P total $(\mu \mathrm{g} / \mathrm{l}$ de $\mathrm{P})$} & \multicolumn{2}{|c|}{$\mathrm{NO}_{2}(\mu \mathrm{g} / \mathrm{de} \mathrm{N})$} & \multirow{2}{*}{$\frac{\text { Secchi }}{(\mathrm{m})}$} \\
\hline & DATES & surface & fond & surface & fond & surface & fond & surface & fond & surface & fond & surface & fond & \\
\hline \multirow{10}{*}{$\begin{array}{l}1 \\
9 \\
9 \\
3\end{array}$} & 20 -janv & 9,7 & 9,6 & 13,2 & 13,2 & 7,3 & 8,2 & 4,0 & 4,0 & 14,0 & 16,0 & 9,0 & 10,0 & 3,5 \\
\hline & 19-févr & 9.7 & 9,7 & 13.3 & 13,3 & 4,9 & 4,8 & 2,1 & 2,3 & 18,6 & 18,5 & 10,0 & 10,0 & 1,9 \\
\hline & 17-mars & 4,0 & 3,0 & 13,2 & 12,2 & 3,8 & 3,2 & 2,1 & 2,3 & 28,4 & 21,6 & 8,0 & 8,0 & 1,8 \\
\hline & $14-\mathrm{avr}$ & 7,0 & 6,0 & 13,0 & 12,5 & 9,4 & 9,1 & 1,0 & 1,2 & 37,4 & 31,0 & 34,0 & 12,0 & 1,4 \\
\hline & 06-mai & 11,5 & 7.0 & 10,8 & 10,2 & 10,8 & 8.5 & 1,3 & 1,7 & 33,0 & 26,6 & 12,0 & 14,0 & 1,0 \\
\hline & 03-juin & 14,0 & 13,0 & 10,2 & 10,0 & 13,5 & 4,8 & 0,5 & 0,5 & 48,0 & 41,0 & 18,0 & 18,0 & 1,1 \\
\hline & VIDANGE & & & & & & & & & & & & & \\
\hline & 14-oct & 14,0 & 13,5 & 10,6 & 10,6 & 4,3 & 3,2 & 4,9 & 5,0 & 33,0 & 22,0 & 36,0 & 41,0 & 2,7 \\
\hline & 04-nov & 11,0 & 10,8 & 11,0 & 11,0 & 9,1 & 8,5 & 5.4 & 5,5 & 22,5 & 20,0 & 23,0 & 30,0 & 2,2 \\
\hline & 15-déc & 5,8 & 6,0 & 12,5 & 12,3 & 4,9 & 6,4 & 5,4 & 5,5 & 25,0 & 60,0 & 16,0 & 10,0 & 2,6 \\
\hline \multirow{11}{*}{4} & 19-janv & 4,6 & 4,2 & 12,6 & 12,5 & 2,0 & 0,3 & 7,6 & 7,4 & 19,0 & 18,0 & 18,0 & 19,0 & 2,6 \\
\hline & 04-inars & 5,5 & 5,5 & 12,5 & 12,6 & 2,2 & 1,1 & 7,8 & 7,9 & 20,6 & 18.8 & 16,0 & 17,0 & 2,5 \\
\hline & 20 -avr & 6,5 & 6,5 & 13,2 & 13,1 & 1,1 & 0,6 & 6,4 & 6,4 & 0,1 & 0,0 & 10,0 & 7,0 & 2,7 \\
\hline & 19-Imai & 11,1 & 8,6 & 11,1 & 11,2 & 2,7 & 1,0 & 5,3 & 6,6 & 16,0 & 9,0 & 11,0 & 11,0 & 4,0 \\
\hline & 13-juin & 16,5 & 11,1 & 9.9 & 9,6 & 1,4 & 0,0 & 4,4 & 5,7 & 29,0 & 13,0 & 16,0 & 58,0 & 7,0 \\
\hline & 22-juil & 22,0 & 12,1 & 9,9 & 3,2 & 9,2 & 0,0 & 3,6 & 5,9 & 15,0 & 12,0 & 24,0 & 5,0 & 5,1 \\
\hline & 30-août & 21,5 & 11,5 & 9,5 & 0,3 & 4,7 & 0,7 & 3,5 & 5,9 & 6,5 & 3,6 & 29,0 & 0,1 & 5,2 \\
\hline & 10-sept & 17,0 & 12,0 & 9,2 & 3,4 & 3,9 & 1,1 & 4,0 & 3,0 & 16,4 & 7,6 & 14,0 & 18,0 & $3 ; 2$ \\
\hline & 05 -oct & 13,0 & 13,0 & 8,5 & 8,5 & 1,9 & 0,0 & 4,7 & 6,3 & 11,0 & 14,4 & 35,0 & 42,0 & 4,1 \\
\hline & 22-nov & 9.0 & 7,5 & 10,0 & 9.8 & 0,0 . & 0,2 & 5,5 & 5,2 & 17.0 & 20,4 & 15,0 & 17,0 & 5,1 \\
\hline & 13-déc & 7,0 & 6,5 & 10,2 & 9,8 & 3,9 & 3,7 & 5,6 & 5,8 & 20,0 & 18.8 & 12,0 & 12,0 & 4,4 \\
\hline
\end{tabular}


L'impact du broutage par le zooplancton est estimé à partir des taux de filtration moyens établis après combinaison de deux méthodes (fluorimétrie et radiotraceur) par Baucheff-Nielsen (1988) sur le même site :

- 1,29 ml/ind./jour pour les petits brouteurs ;

- 2,34 ml/ind./jour pour les grands brouteurs ;

- 2,03 ml/ind./jour pour les herbivores.

Les Rotifères, généralement peu représentés à Pareloup, n'ont pas été pris en considération dans cette étude.

Simultanément, un prélèvement pour l'analyse de la composition biochimique du plancton est réalisé à l'aide d'un filet de $45 \mu \mathrm{m}$ vide de maille tracté entre 0 et 10 mètres de profondeur. Sous l'effet de la traction et du colmatage progressif, cette méthode permet le recueil d'un concentré contenant tout le zooplancton et la presque totalité du phytoplancton. L'échantillon de plancton est déshydraté dans une étuve à vide Heraeus sur chlorure de calcium à température ambiante.

Six réplicats par mois ont été analysés, les lipides totaux sont déterminés par gravimétrie après extraction selon la méthode de Folch et al. (1957), les protéines brutes par la technique de Kjeldhal ( $\mathrm{N}$ total x 6,25).

La variabilité des données en fonction des mois a été estimée par une ANOVA à un critère de classification (Systat, Wilkinson 1989). Lorsque les variations sont significatives au seuil de $5 \%$, l'analyse est complétée par un test de comparaison multiple des moyennes (Tukey-HSD).

\section{Résultats}

La première période de l'étude (janvier - juin 1993) est caractérisée par une hausse des concentrations en chlorophylle a et en phosphore total accompagnée d'une diminution de la profondeur du disque de Secchi (Tableau 1), indiquant une tendance à l'eutrophisation du milieu. Toutefois, aucune réduction conséquente des teneurs en oxygène dissous n'a été observée, de même, la température alors enregistrée n'indique pas la mise en place d'une stratification thermique.

Durant la seconde période (octobre 1993 - décembre 1994) on observe une diminution de la concentration en chlorophylle a, en particulier au printemps 1994 . Simultanément, la profondeur du disque de Secchi augmente pour atteindre une valeur maximale de 7 mètres le 13 juin 1994. En hiver et au printemps 1994 les valeurs des concentrations en silice sont comparativement plus élevées qu'en 1993. Les relevés de température et d'oxygène dissous indiquent la mise en place d'une stratification de mai à septembre 1994 (Tableau 1).
Les crustacés planctoniques sont essentiellement représentés par Daphnia longispina (O.F. .Müller, 1785), Ceriodaphnia quadrangula (O.F. Müller, 1785), Bosmina longirostris (O.F. Müller, 1785) pour les Cladocères et par Cyclops vicinus vicinus (Ulianine, 1875), Acanthocyclops robustus (G.O. Sars, 1863), et Eudiaptomus vulgaris (Schmeil, 1896) pour les Copépodes.

L'évolution saisonnière de la densité (Fig. 2a) et de la biomasse (Fig. 3) est envisagée pour les quatre principales familles (Daphniidae, Bosminidae, Cyclopidae et Diaptomidae) ; la structure du peuplement microcrustacés a été étudié pour les six espèces dominantes de la retenue (Fig. 2b).

\subsection{Densité et évolution de la structure du peuple- ment}

Durant la phase d'abaissement du plan d'eau, on observe à partir du mois de février une augmentation régulière de la densité totale qui atteint un maximum de 22,5 ind./litre en mai 1993 (Fig. 2a). Le peuplement hivernal est constitué d'une forte proportion de Cladocères avec une prédominance de $B$. longirostris. Hormis une légère hausse de l'effectif des Bosminidae ou des Daphniidae, respectivement en avril et mai, on note surtout un développement des Cyclopidae. Les densités maximales des adultes d'A. robustus et des copépodites de cyclopides ainsi que la chute de l'effectif des Cladocères, entraînent une domination des Cyclopidae de l'ordre de $70 \%$ en juin 1993 (Fig.2b). Le Diaptomide $E$. vulgaris, peu représenté, est absent du dernier relevé précédent la vidange. De même, $C$. quadrangula n'apparaît pas dans les prélèvements durant la période d'abaissement du plan d'eau.

Avec le début de la remise en eau du lac, à l'automne 1993 on enregistre la valeur maximale de densité de toute la période d'étude $(66,7$ ind./l, le 13 octobre 1993), Copépodes et Cladocères sont alors en proportions égales. Le peuplement est essentiellement constitué par des nauplii et copépodites de Cyclopides et par C. quadrangula. A partir de novembre 1993, et durant tout l'hiver 1994, la forte production de jeunes stades de Cyclopidae et l'augmentation sensible des adultes d'A. robustus et de $C$. vicinus, entraîne la prédominance des Cyclopidae.

L'année 1994 est caractérisée par deux pics d'abondance maximale comparables, l'un au printemps (mai), l'autre en automne (septembre), séparés par une phase de déclin estival.

Au printemps, le développement de $D$. longispina contribue à l'importante domination des Cladocères qui représentent environ $90 \%$ des microcrustacés en mai 1994. Le déclin estival des zooplanctontes s'ac- 

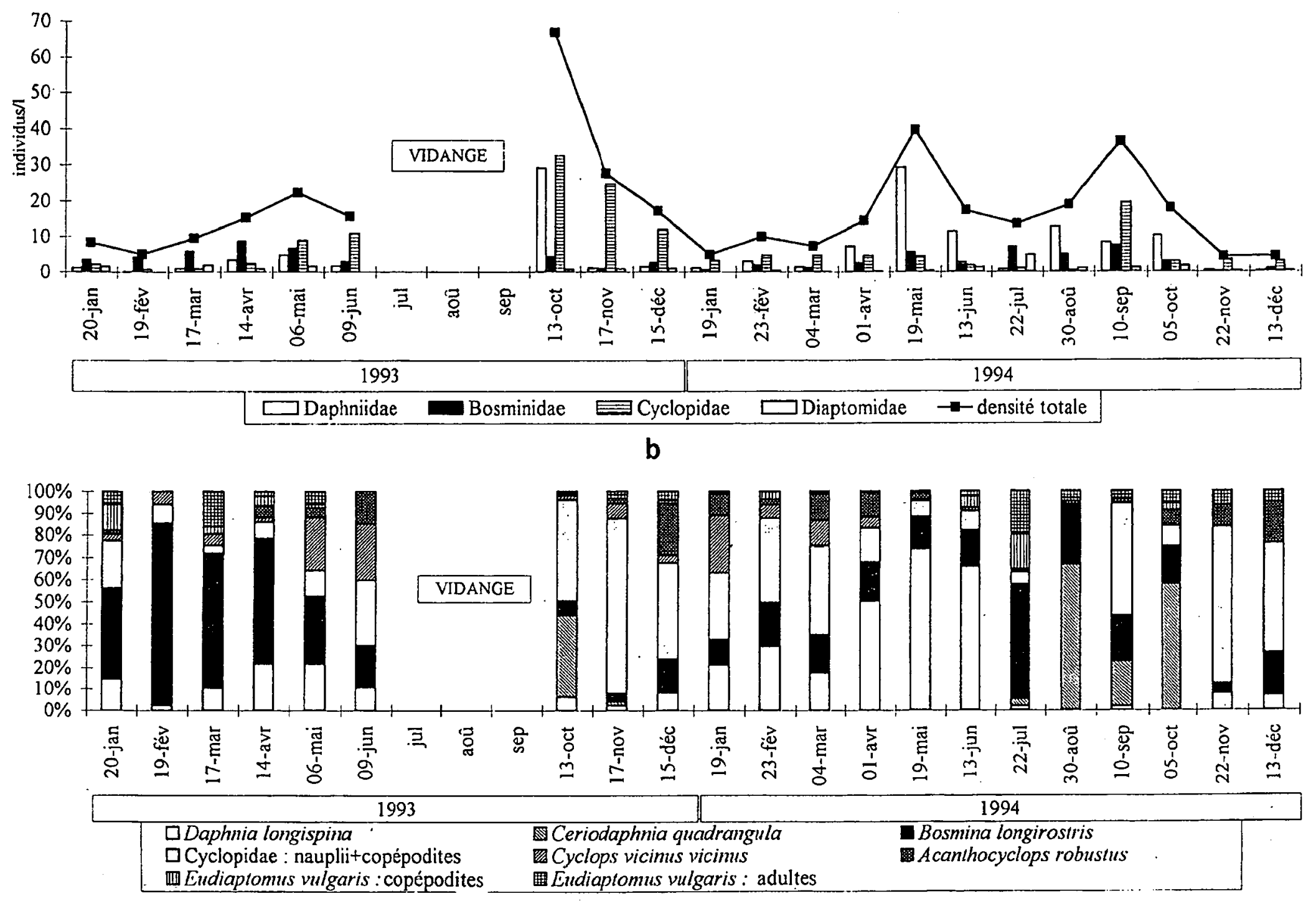

Fig. 2. Evolution de la densité des microcrustacés planctoniques (a), et composition relative (b) en \% de la densité.

Fig. 2: Evolution of the planktonic microcrustacean density (a) and relative composition (b) in $\%$ of density.

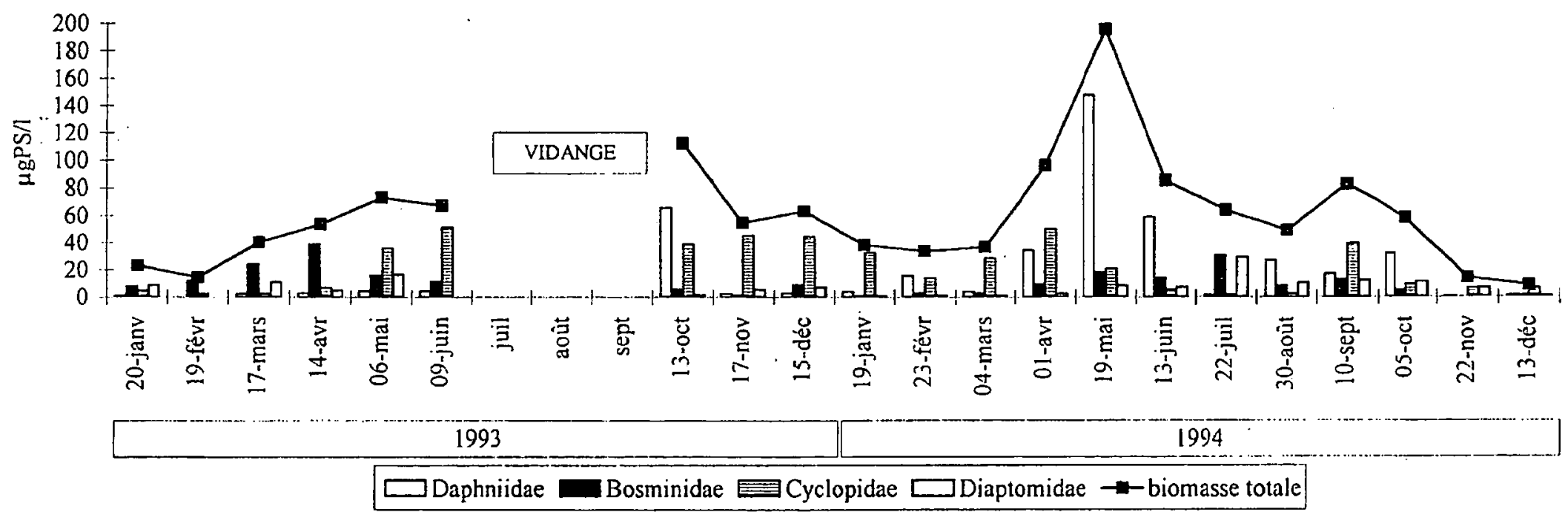

Fig. 3. Evolution de la biomasse des microcrustacés planctoniques.

Fig.3. Evolution of the planktonic microcrustacean biomass. 
compagne d'une diversification du peuplement due à l'apparition de $C$. quadrangula et au développement de $E$. vulgaris $(4,8$ ind./litre, le 22 juillet) et $B$. longirotris ( 7 ind./litre, le 22 juillet). Le pic d'abondance automnal est caractérisé par une hausse des copépodites de cyclopide constituant en septembre $50 \%$ de la communauté zooplanctonique avec 19,58 ind./litre. En fin d'automne début de l'hiver, malgré de faibles densités, les Copépodes restent majoritaires avec une contribution d'environ $75 \%$. Toutefois, il faut noter l'absence de $C$. vicinus dans les prélèvements des cinq derniers mois étudiés.

\subsection{Biomasse}

Si généralement l'évolution de la biomasse est similaire à celle de la densité, on peut observer des discordances en raison du fort écart pondéral existant entre les divers stades de développement des organismes et en particulier chez les Copépodes.

Durant la phase de vidange, les variations de la biomasse totale sont identiques à celles des densités, la valeur maximale est notée en mai 1993 avec 73,4 $\mu \mathrm{g}$ PS/litre (Fig. 3). En revanche, dès la remise en eau et durant l'année 1994, il apparaît des différences entre les deux paramètres. Ainsi, l'augmentation de la biomasse en décembre 1993 concomitante à une diminution de la densité est due à la forte contribution des $\mathrm{Cy}$ clopidae à cette période. De même, l'important effectif observé en octobre 1993 ne correspond pas à la plus forte valeur de biomasse ; le maximum pour les deux années $(195 \mu \mathrm{g}$ PS/l) est enregistré en mai 1994. Pour des périodes identiques, les biomasses sont supérieures après octobre 1993. Enfin, on peut noter qu'en 1994 la biomasse automnale ne représente que la moitié de sa valeur printanière malgré des pics d'abondance comparables. Ceci peut être expliqué par la prédominance des Daphniidae au printemps et celle des nauplii et copépodites de Cyclopides d'un faible poids individuel en automne.

\section{3. Évolution des groupes de consommateurs et impact sur la communauté phytoplanctonique}

L'évolution saisonnière des proportions de chaque groupe de consommateurs zooplanctoniques permet d'appréhender leur impact potentiel sur la communauté phytoplanctonique (Fig. 4a et 4b).

Au cours de l'hiver et au début du printemps 1993, le peuplement est dominé numériquement par les petits brouteurs avec une proportion maximale de $70 \%$ en février. En revanche, ils ne représentent que 10 à $20 \%$ de la densité en 1994, hormis au mois d'août où ils dominent à nouveau les autres groupes. Les grands brou- teurs sont majoritaires d'avril à juin 1994. Les herbivores, peu nombreux, ont une contribution moyenne annuelle inférieure à $10 \%$. En automne et durant l'hiver 1994, les carnivores (copépodites IV à adultes de Cyclopidae) constituent le groupe le plus important ; ils peuvent représenter plus de $80 \%$ du peuplement au mois de novembre quelle que soit l'année considérée.

L'évolution saisonnière de la biomasse des différents groupes de consommateurs (Fig. 4b), est globalement similaire à celle décrite pour les densités. Cependant, durant l'hiver 1993, la prédominance des petits brouteurs disparaît au profit de celle des grands brouteurs.

En 1994, quoique plus marquée, l'alternance des phases de biomasse de chaque catégorie reste semblable à celle notée pour les densités.

L'important poids individuel des herbivores confère au groupe une contribution pondérale importante en juillet et en novembre 1994 qui n'apparait pas en terme de densité.

Les taux de filtration moyens individuels calculés pour les différents groupes par Baucheff-Nielsen (1988) ont été multipliés par la densité de chaque groupe afin d'estimer d'une part, le volume moyen d'eau filtrée potentiellement par jour et par groupe exprimés en pourcentage du volume d'eau du lac (Fig. 5a) et d'autre part, la contribution respective de chaque groupe au taux de filtration potentiel (Fig. 5b).

En hiver et au printemps 1993, l'impact des petits brouteurs est prépondérant. Le volume moyen d'eau filtrée est de l'ordre de $2 \%$ par jour. En revanche, ce sont les grands brouteurs avec $67 \%$ du volume potentiel d'eau filtrée qui constituent d'octobre à juin 1994 les principaux régulateurs de la population algale. $\mathrm{Au}$ printemps 1994, le développement des grands brouteurs entraîne une hausse du volume moyen filtré par jour qui atteint une valeur maximale de $8 \%$ en mai. En été, l'influence de ce groupe diminue au profit des herbivores Calanoïdes en juillet ( $45 \%$ ) et des petits brouteurs en août $(56 \%)$. Les faibles effectifs et l'importante proportion de copépodites enregistrés en décembre 1994 entraînent des contributions voisines pour les trois types de filtreurs. Le volume moyen d'eau filtrée est alors très faible (moins de $1 \%$ par jour).

\subsection{Composition biochimique du plancton}

La composition biochimique du plancton est significativement différente pour les deux années étudiées (Fig. 6).

Les teneurs en lipides, voisines de $7,9 \%$ du poids sec en 1993, ont tendance à augmenter au printemps ; 

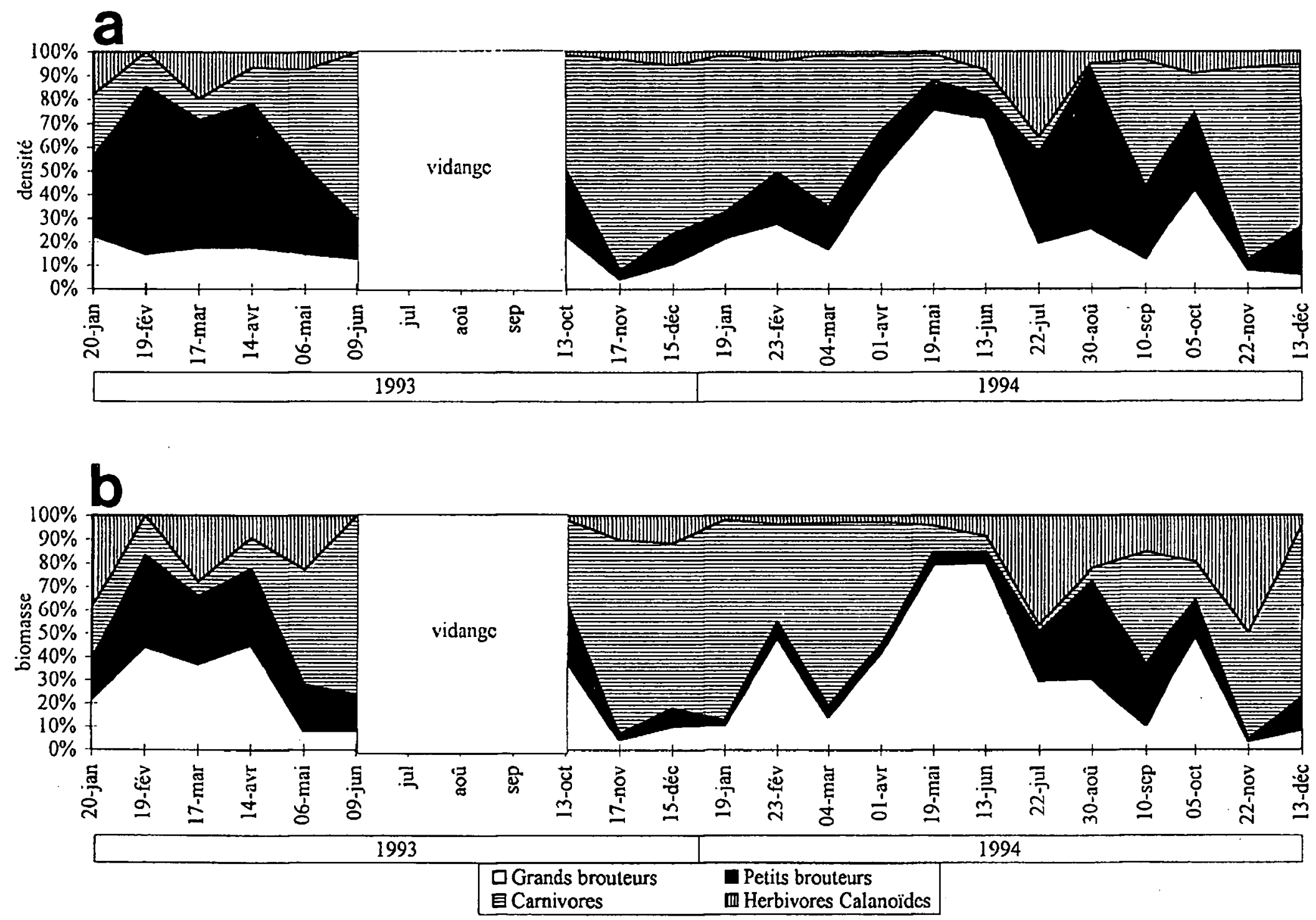

Fig. 4. Composition relative de la densité (a) et de la biomasse (b) des différentes catégories de consommateurs.

Fig. 4. Relative composition of the density (a) and biomass (b) of the different zooplankton grazing classes.

elles atteignent un maximum de $12,7 \%$ du poids sec en mai. En 1994, la valeur moyenne annuelle est de $9,3 \%$, avec un pic important $(11,8 \%)$ en février. Hormis une hausse en juillet et une diminution significative en fin d'automne, le pourcentage de lipide reste stable (8 à $10 \%$ du poids sec) tout au long de l'année 1994.

Les protéines présentent des évolutions inverses pour les deux années entre les mois de janvier et de juin. En 1993, les teneurs moyennes sont de l'ordre de $27 \%$ du poids sec et augmentent de l'hiver au printemps. En 1994, pour la même période, la valeur moyenne est de $47,8 \%$ et diminue de l'hiver au printemps 1994. En été, on observe une hausse des protéines planctoniques. L'automne est caractérisé par des teneurs similaires quelle que soit l'année, cependant on enregistre une baisse plus importante en décembre 1994 avec un minimum annuel de $23,5 \%$ du poids sec.

\section{Discussion}

Dans les conditions de changement drastique du milieu, et notamment l'importante variation de la biomasse pisciaire à l'hectare pendant et après la vidange, on pouvait supposer des modifications de la structure, de la biomasse et de la densité de la communauté zooplanctonique.

McQueen \& Post (1988) ont montré sur le lac StGeorges, qu'une biomasse pisciaire supérieure à 100 kg.ha ${ }^{-1}$ est nécessaire pour observer un effet négatif sur une population de daphnies. Lazzaro et al. (1992) indiquent que la quantité de poissons joue un rôle plus important dans la régulation des communautés planctoniques que le type de planctonophage considéré.

Dans le lac de Pareloup, avant la vidange, le gardon et la perche dominent numériquement la communauté pisciaire. Des études du régime alimentaire de ces deux Téléostéens, ont montré la prédominance du zoo- 

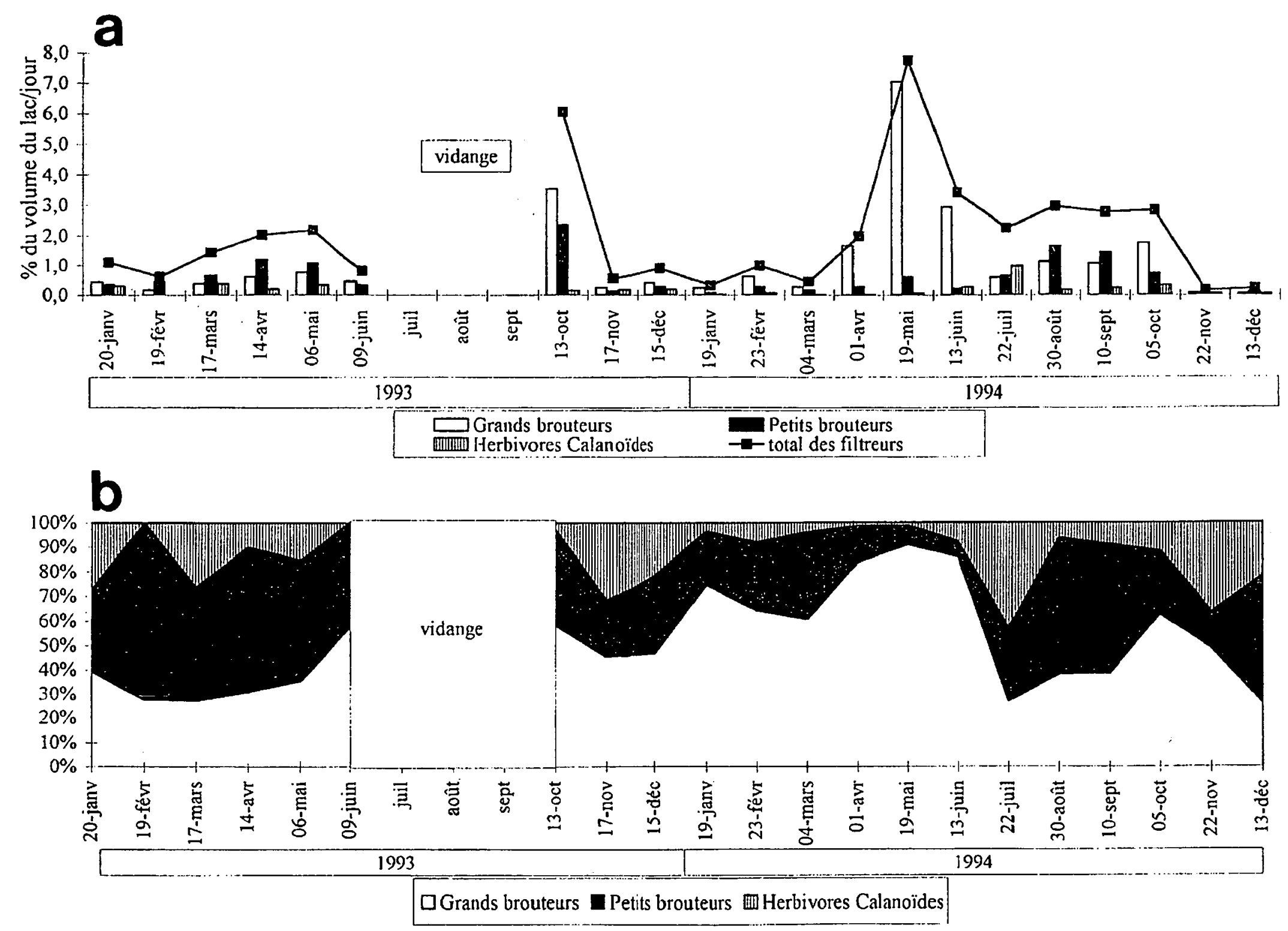

Fig. 5. Taux de filtration potentiel (a) et contribution des trois catégories de brouteurs.

Fig. 5. Potential filtering rate (a) and contribution (b) of the three filter feeding classes.

plancton dans l'alimentation des alevins au printemps et au début de l'été (Richeux et al. 1992, Dubois et al. 1994). L'évolution saisonnière du spectre alimentaire des adultes est caractérisée, en particulier chez le gardon, par une augmentation de la proportion de zooplancton au printemps (Richeux et al. 1994b). D'autres auteurs signalent l'importance des Cladocères dans la nutrition de ces poissons (Hammer 1985, Townsend \& Perrow 1989, Jamet et al. 1990, Reyes-Marchand 1993).

L'impact des poissons zooplanctophages se traduit généralement par une diminution de la taille moyenne des individus (Brooks \& Dodson 1965) et par une prédation sélective sur les grands brouteurs (Daphnia) entraînant une domination des petits brouteurs (Bosmina) (Shapiro \& Wright 1984, He et al. 1994, Visman et al. 1994).

Certains travaux mettent en évidence l'existence d'effets directs ou indirects des populations planctonophages sur le développement des Cladocères. Vanni
(1986) et DeVries \& Stein (1992) signalent l'importance de l'impact des poissons sur la structure du peuplement phytoplanctonique et ses conséquences indirectes sur les zooplanctontes. Gliwicz (1994) montre qu'une diminution de la densité du zooplancton résulte de la combinaison entre un effet direct (mortalité par prédation) et des effets indirects (diminution des taux de croissance et de reproduction).

Une importante proportion de poissons planctonophages peut induire une hausse des Cyclopidae dans le milieu (Rudstam et al. 1993). Ce phénomène pèrmet une compensation de la perte de la biomasse cladocérienne consécutive à une forte prédation. Toutefois, Maier (1996) signale une augmentation des Cyclopidae avec le niveau trophique du lac. La tendance à l'eutrophisation observée à Pareloup peut en partie expliquer la domination de ce groupe en juin 1993. Santer (1993) indique l'existence au printemps d'une prédation des adultes de cyclopides sur les daphnies pour éviter une possible compétition trophique des jeunes 

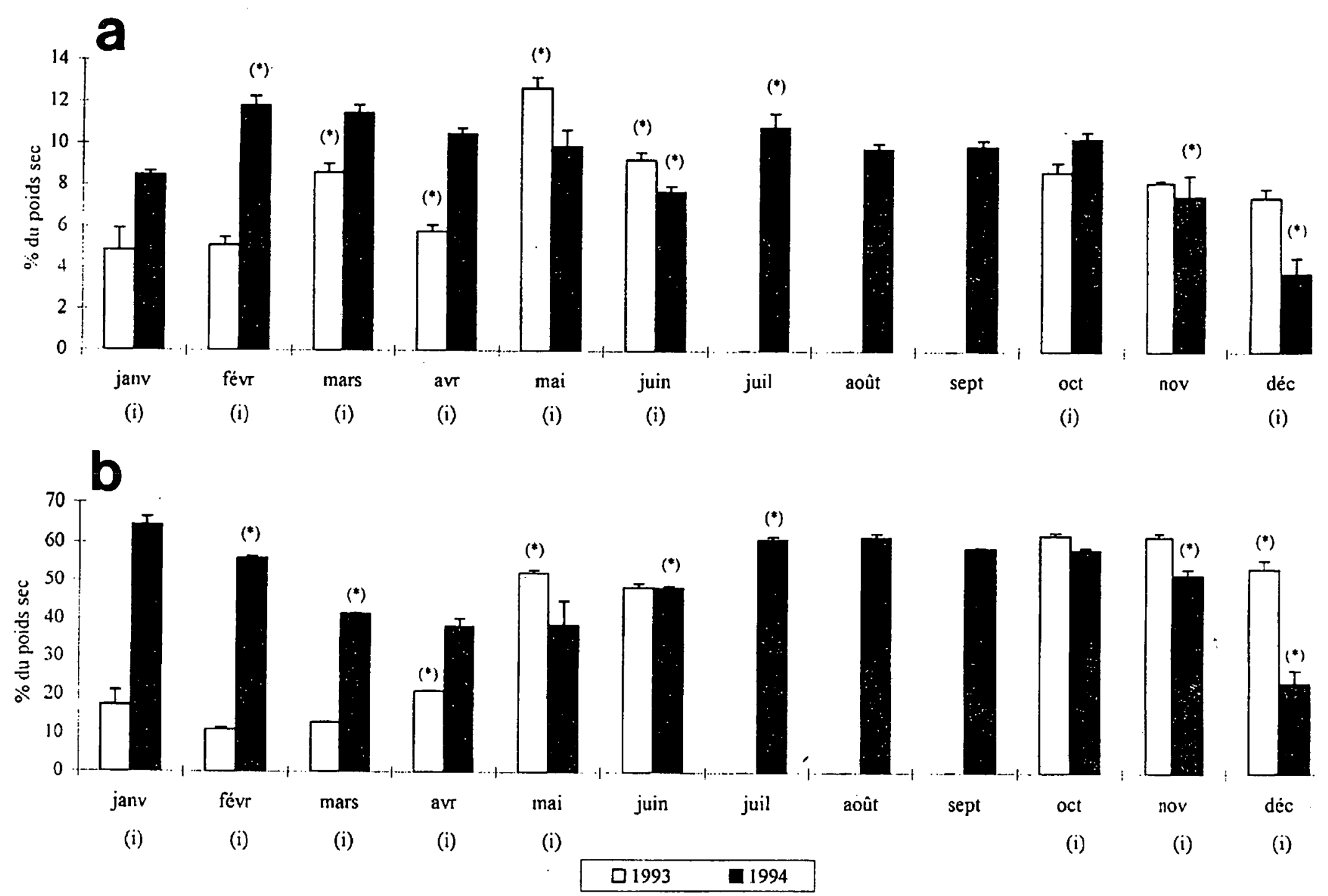

Fig. 6. Teneurs en lipides (a) et protéines (b) en pourcentage du poids sec du plancton.

Les valeurs présentées sont des moyennes $+/$ - écart-types ; (i) valeurs significativement différentes entre les deux années $(p(0,05) ;(*)$ indique une différence significative par rapport au mois précédent ( $p(0,05)$ (janvier-juin 1993 : d'après Ferroni et al. 1996).

Fig. 6. Levels of lipids (a) and proteins (b) of plankton (in \% of dry weight).

Values given are means $+/$ - SD; (i) significant differences between the two years $\left(p(0,05) ;\left(^{*}\right)\right.$ denotes a significant difference comparing to previous month ( $p(0,05)$ (January-June 1993 : from Ferroni et al. 1996).

stades de ces deux groupes. Bien représentés, les adultes de $C$. vicinus et d'A. robustus contribuent probablement au déclin des Daphniidae observé en mai et juin 1993 à Pareloup.

L'export d'organismes zooplanctoniques lors de la diminution du volume d'eau peut constituer un facteur prépondérant dans la modification de la structure du peuplement. Dans une retenue des Pyrénées, Rey (1988) a montré que la quantité et la nature du zooplancton exporté à l'exutoire lors d'une vidange dépend des migrations verticales, de la hauteur de la prise d'eau et du temps de génération de l'espèce considérée. L'absence d'une stratification thermique réduit les variations verticales de la répartition du zooplancton. L'eau soutirée à un niveau hypolimnique lors de la vidange de Pareloup n'a probablement pas entraîné d'importantes modifications de la concentration des Crustacés.
Les variations de la structure du peuplement microcrustacé - diminution des grands brouteurs, augmentation des petits brouteurs et des copépodes Cyclopidae - observées à Pareloup au cours de la phase de vidange témoignent de la prépondérance des effets descendants de type «prédateurs-proies» essentiellement provoqué par une forte concentration des prédateurs et en particulier des poissons planctonophages.

Après la vidange, la biomasse pisciaire ainsi que la proportion de planctonophages dans le milieu ont considérablement diminué.

Selon le schéma de «cascade trophique» proposé par Carpenter \& Kitchell (1993), une baisse de l'abondance des planctonophages entraîne une augmentation du nombre et de la taille du zooplancton brouteur et, par conséquent, une chute de la biomasse phytoplanctonique ; la phase d'eau claire est alors prolongée dans le temps et les concentrations en chlorophylle a dimi- 
nuent. Seda \& Duncan (1994) ont observé ce phénomène dans plusieurs réservoirs d'eau londoniens : la grande daphnie (Daphnia magna) domine le peuplement Crustacés et son impact se traduit par la diminution du phytoplancton dans ces milieux. De même, des manipulations du stock pisciaire visant à réduire les poissons planctonophages et à augmenter les piscivores engendrent des modifications de la structure de la population zooplanctonique se traduisant par une hausse de la contribution des grands brouteurs. Il s'ensuit généralement une baisse de la quantité de phytoplancton correspondant à une intensification de la pression de broutage (Shapiro \& Wright 1984 , Vanni et al. 1990, Christoffersen et al. 1993).

L'exploitation de la biomasse algale est généralement maximale au printemps (van Donk 1991) et peut engendrer, en été, des conditions nutritionnelles défavorables au développement des Crustacés. Ainsi, Larsson et al. (1985) ont montré, suite à l'élimination de la fraction consommable, que l'apparition d'espèces algales non consommables (Cyanophycées) contribue au déclin estival de Daphnia longispina.

Les taux de filtration pour une espèce considérée varient en fonction de nombreux facteurs tels que : les concentrations de l'aliment (Muck \& Lampert 1980, Gulati et al. 1991), la température, le moment de la journée (Haney 1985), l'état nutritionnel ou encore la qualité de la nourriture (Schindler 1971). Bien qu'il faille être prudent quant à la validité des taux de filtration moyens utilisés dans cette étude, ils rendent compte de l'augmentation de la pression de broutage au printemps 1994. Le pourcentage du volume d'eau du lac filtrée par jour est multiplié par quatre à cette saison après la vidange de la retenue.

De même, les faibles concentrations en chlorophylle a (Tableau 1) observées au printemps 1994 dans le lac de Pareloup, témoignent d'une forte pression de broutage de Daphnia longispina. L'important taux de chlorophylle a, relevé en juillet, résulte certainement du développement en quantité d'une algue non consommable (Gonyostomum semen) précédemment observé dans la retenue à cette saison (Le Cohu et al. 1991). L'absence de la daphnie indique la mise en place d'un peuplement algal défavorable à un essor estival de cette espèce. De plus, l'apparition en fin d'été des petits brouteurs (B. longirostris, $C$. quadrangula) ainsi que les valeurs élevées de la profondeur du disque de Secchi, attestent de l'important impact printanier de la population zooplanctonique sur la communauté phytoplanctonique. Plusieurs auteurs signalent l'existence d'une succession des groupes zooplanctoniques afin d'éviter une possible compétition trophique (Garnier
\& Lescher-Moutoué 1984, Edmonson 1985, De Bernardi et al. 1988, Francisco \& Rey 1994).

L'accroissement des Cyclopidae observé les années précédentes au début de l'été (Francisco \& Rey 1994) n'apparaît pas en 1994. La faible prédation des poissons permet au printemps un développement maximal de $D$. longispina. L'exploitation intensive du milieu par la daphnie entraîne une réduction de la disponibilité phytoplanctonique défavorable aux Cyclopidae (Santer \& Lampert 1995, Maier 1996). En septembre, la diminution des grands brouteurs autorise le développement automnal de ce groupe. La dominance hivernale des cyclopides est due aux faibles densités des Cladocères dans le milieu plutôt qu'à un réel essor de ces copépodes à cette saison.

L'évolution de la structure du peuplement microcrustacé observée en 1994, témoigne d'une réduction de la pression de prédation. Le développement du zooplancton est alors sous l'influence de facteurs de type ascendant (disponibilité phytoplanctonique) en particulier au printemps et en été.

Bien que le plancton constitue une ressource alimentaire importante pour les poissons, peu de travaux font état des variations saisonnières de la composition biochimique du phytoplancton et du zooplancton en milieu naturel (Pourriot \& Leborgne 1970, Vijverberg \& Frank 1976, Ferroni 1996).

Les teneurs en lipides évaluées chez quelques algues maintenues en culture varient en fonction de l'espèce, de son stade de développement et des conditions de culture (Tedesco \& Duerr 1989, Harrisson et al. 1990, Chen \& Johns 1991, Sicko-Goad \& Andresen 1991). Concernant le zooplancton, les lipides constituent une importante réserve énergétique. Les variations sont liées au cycle de développement des espèces (mobilisation des lipides maternels vers les oeufs), à la quantité et à la qualité du phytoplancton ingéré (Schindler 1971, Infante \& Litt 1985, Tessier \& Goulden 1982, Ahlgren et al. 1990). L'élévation de la température du lac peut entraîner une hausse du pourcentage de lipides dans le zooplancton (Kuosa \& Gyllenberg 1989). L'ensemble de ces travaux apparaît difficilement comparable à ceux réalisés sur une fraction planctonique (Phytoplancton + Zooplancton) prélevée en milieu naturel. Comme le signalent Ferroni et al. (1996), dans le lac de Pareloup, les variations des teneurs en lipides du plancton particulaire dépendent essentiellement des proportions de zooplancton et de phytoplancton ainsi que de leur pourcentage de lipides respectif. Ainsi, en hiver et au début du printemps, la domination des Diatomées entraîne des valeurs faibles $(5$ à $8 \%$ du poids $\mathrm{sec})$. Au début de l'été, succède à la disparition des 
Diatomées une population algale plus diversifiée ; la biomasse et la charge lipidique du zooplancton sont alors maximales et on observe, à cette saison, une élévation du pourcentage de lipides planctoniques.

En hiver et au début du printemps 1994, les teneurs en lipides sont supérieures à celles notées les années précédentes (Ferroni et al. 1996). Les concentrations en silice élevées à cette période (Tableau 1), laissent supposer une moindre utilisation par les Diatomées. De même, les faibles valeurs de chlorophylle a témoignent d'une diminution de la biomasse algale. L'augmentation de la contribution zooplanctonique au plancton particulaire consécutive à la diminution du phytoplancton et en particulier des Diatomées, pourrait expliquer les fortes teneurs en lipides relevées en 1994. De plus, le zooplancton est essentiellement constitué de Cyclopidae, groupe dont le pourcentage de lipides est le plus élevé à cette période (Ferroni et al. 1996).

Le pourcentage de protéines d'une population phytoplanctonique dépend des espèces dominantes présentes dans la fraction analysée. Il peut être de l'ordre de $20 \%$ du poids sec pour une population dominée par les Diatomées (Behrendt 1990) et de $40 \%$ pour un peuplement essentiellement composé de Pyrrhophycées (Khan \& Siddiqui 1971). Ahlgren et al. (1992), indiquent, dans le cas de culture, qu'il peut atteindre $50 \%$ chez certaines Chlorophycées et chez des Dinoflagellés. Les teneurs en protéines du zooplancton sont élevées quel que soit le stade de développement (Baudoin \& Ravera 1972, Mc Kee \& Knowles 1987). Selon Yurkowski \& Tabachek (1979), il varie avec la saison et oscille entre 29 et $74 \%$ pour les Cladocères et 38 à $72 \%$ pour les Copépodes, les valeurs les plus faibles étant relevées en hiver.

Les faibles teneurs notées durant l'hiver 1993 correspondent à une population planctonique dominée par les Diatomées (Ferroni et al. 1996). En revanche, comme pour les lipides, les valeurs les plus élevées du pourcentage de protéines enregistrées pendant l'hiver 1994 pourraient provenir de l'accroissement de la contribution du zooplancton et d'une plus grande diversité spécifique du peuplement algal.

Globalement, la valeur nutritive du plancton est supérieure après la vidange, notamment durant l'hiver et le début du printemps. Les modifications de la structure du peuplement zooplanctonique et leurs incidences sur la communauté phytoplanctonique semblent expliquer ces variations. Il faut cependant noter que pour une saison donnée, la valeur nutritive du plancton peut être élevée mais les quantités disponibles faibles. Ferroni et al. (1996), soulignent l'importance de ce phénomène pour la croissance des poissons peuplant la retenue.
Les variations de la biomasse étudiées pour chaque groupe phytoplanctonique et zooplanctonique doivent compléter ces résultats et nous permettre de calculer les quantités réellement disponibles de protéines et de lipides du plancton au cours de cycles annuels. Ceci pourrait peut être nous aider à préciser les capacités d'accueil des milieux lacustres et leur évolution dans le temps à partir de la remise en eau et du suivi de la population pisciaire.

\section{Références}

Ahlgren G., Gustafsson I.B. \& Boberg M. 1992. - Fatty acid content and chemical composition of freshwater microalgae. $J$. Phycol., 28 : 37-50.

Ahlgren G., Lundstedt L, Brett M. \& Forsberg C. 1990. - Lipid composition and food quality of some freshwater phytoplankton for cladoceran zooplankters. J. Plankton Research, 12 (4) : 809818.

Baucheff-Nielsen P. 1988. - Contribution à l'écologie du zooplancton du lac de Pareloup. Thèse $3^{\text {eme }}$ cycle. Universite Paul Sabatier, Toulouse III : $104 \mathrm{p}$.

Baudouin M.F. \& Ravera O. 1972. - Weight, size and chemical composition of some freshwater zooplankters : Daphnia hyalina (Leydig). Limnol. Oceanogr., 17 : 645-649.

Behrendt H. 1990. - The chemical composition of phytoplankton and zooplankton in an eutrophic shallow lake. Arch. Hydrobiol. $118(2): 129-145$

Bottrell H.H., Duncan A., Gliwicz Z.W., Grygierek E., Herzig A., Hillbricht-Ilkowska A., Kurusawa H., Larsson P. \& Weglenska T. 1976. - Contribution from the plankton ecology group. A review of some problems in zooplankton production studies. Norw. J. Zool, $24: 419-456$.

Brooks J. \& Dodson I. 1965. - Predation, body size and composition of plankton. Science, $150: 28-35$.

Carpenter S.R., Kitchell J.F. (Eds) 1993. - The trophic cascade in lakes. Cambridge University Press, New York : $350 \mathrm{p}$

Carpenter S.R., Kitchell J.F. \& Hodgson J.R. 1985. - Cascading Trophic Interactions and Lake Productivity. BioScience., 35 (10): 634-639.

Carvalho L. 1994. - Top-down control of phytoplankton in a hypertrophic lake : Little Mere (England). Hydrobiologia, 275/276: 53-63.

Chen F. \& Johns M.R. 1991. - Effect of $\mathrm{C} / \mathrm{N}$ ratio and aeration on the fatty composition of heterotrophic Chlorella sorokiniana. $J$. Appl. Phycol., 3 : 203-209.

Christoffersen K., Riemann B., Klysner A. \& S(ndergaard M. 1993. - Potential role of fish predation and natural populations of zooplankton in structuring a plankton community in eutrophic lake water. Limnol. Oceanogr. 38 (3) : 561-573.

De Bernardi R., Giussani G. \& Manca M. 1988. - Seasonal evolution of Cladocera in Lago Maggiore (N. Italy) as influenced by environnemental parameters. Verh. Internat. Verein. Limnol., 23 : 510-534.

DeVries D.R. \& Stein R.A. 1992. - Cómplex interactions between fish and zooplankton : quantifying the role of an open-water planktivore. Can. J. Fish. aquat. Sci., 49 : 1216-1227.

Donk E. van 1991. - Changes in community structure and growth limitation of phytoplankton due to top-down food-web manipulation. Verh. Internat. Verein. Limnol., 24 : 773-778.

Dubois C., Richeux C., Tourenq J.N. \& Lejolivet C. 1994. - Régime et comportement alimentaire du gardon (Rutilus rutilus, L.) et de la perche (Perca fluviatilis, L.) de la retenue de Pareloup. I. Les alevins. Hydroécol. Appl., 6 (1/2) : 227-242. 
Dumont H.S., Van de Velde I. \& Dumont S. 1975. - The dry weight estimate of biomass in selection of Cladocera, Copepoda and Rotifera from plankton periphyton and benthos of continental waters. Oecologia., $19: 75-97$.

Edmonson W.T. 1985. - Reciprocal changes in abundance of Diaptomus and Daphnia in lake Washington. Arch. Hydrobiol. Beih. Ergebn. Limnol., 21 : 475-481.

Evans M.S. 1990. - Large-lake responses to declines in the abundance of a major fish planktivore - the Lake Michigan example. Can. J. Fish. Aquat. Sci., 47 : 1738-1754.

Ferroni J.M. 1996. - Caractéristiques morphophysiologiques et composition somatique de la perche (Perca fluviatilis L.) et du gardon (Rutilus rutilus L.) dans un lac de retenue : Pareloup (France). Variations saisonnières et relations avec la valeur nutritive du plancton. Thèse d'Université, Université Paul Sabatier, Toulouse III : $92 \mathrm{p}$.

Ferroni J.M., Francisco P., Parent J.P. \& Tourenq J.N. 1996. — Evolution saisonnière de la composition biochimique de deux Téléostéens (Perca fluviatilis, L., Rutilus rutilus, L.) et du plancton dans la retenue de Pareloup (Aveyron). Annls. Limnol., 32 (1) : 63-76.

Folch J., Lees M. \& Stanley G.H.S. 1957. - A simple method for the isolation and purification of total lipids from animal tissues. $J$. Biol. Chem., 226 : 497-509.

Francisco P. \& Rey J. 1994. - Etude du peuplement zooplanctonique de la retenue de Pareloup (Aveyron, France). Hydroécol. Appl., $6(1 / 2):$ 175-196.

Garnier J. \& Lescher-Moutoué F. 1984. - Successions saisonnières phyto-zooplanctoniques dans un écosystème lacustre peu profond (lac de Créteil, France). Verh. Internat. Verein. Limnol., 22 : 9961002.

Gliwicz Z.M. 1994. - Relative significance of direct and indirect effetcs of predation by planktivorous fish on zooplankton. Hydrobiologia, 272 : 201-210.

Gulati R.D., Vuick C., Siewertsen K. \& Postema G. 1991. - Clearance rates of Bosmina species in response to changes in trophy and food concentration. Verh. Internat. Verein. Limnol., 24 : 745750.

Hammer C. 1985. - Feeding behaviour of roach (Rutilus rutilus, L.) larvae and the fry of perch (Perca fluviatilis, L.) in lake Lankau. Arch.Hydrobiol., 103 (1) : 61-74.

Haney J.F. 1985. - Regulation of Cladoceran filtering rates in nature by body size, food concentration, and diel feeding patterns. Limnol. Oceanogr., 30 (2) : 397-411.

Harrisson P.J., Thompson P.A. \& Calderwood G.S. 1990. - Effects of nutrient and light limitation on the biochemical composition of phytoplankton. J. Appl. Phycol., 2 : 45-56.

Havens K.E. 1993. - Responses to experimental fish manipulations in a shallow, hypereutrophic lake : the relative importance of benthic nutrient recycling and trophic cascade. Hydrobiologia, 254 : 73-80.

He X., Scheurell M.D., Soranno P.A. \& Wright R.A. 1994. - Recurrent response patterns of zooplankton community to whole-lake fish manipulation. Freshwat. Biol., 32 : 61-72.

Infante A. \& Litt A.H. 1985. - Differences between two species of Daphnia in the use of 10 species of algae in Lake Washington. Limnol. Oceanogr., $30: 1053-1059$.

Jamet J.L., Gres P., Lair N. \& Lasserre G. 1990. — Diel feeding cycle of roach (Rutilus rutilus, L.) in eutrophic Lake Aydat (Massif Central, France). Arch. Hydrobiol., 118 (3) : 371-382.

Khan J.A. \& Siddiqui A.Q. 1971. - Water, nitrogen and phosphorus in freshwater plankton. Hydrobiologia, 37 (3) : 531-536.

Kuosa H. \& Gyllenberg G. 1989. - Lipid content and utilization of lipids in planktonic copepods in lake Pääjärvi, southern Finland. Hydrobiologia, $171: 215-222$.
Larsson P., Andresen S., Børsheim Y., Jakobsen P. \& Johnsen G. 1985. - Individual growth of Daphnia longispina in the summer decline phase of the population. Arch. Hydrobiol. Beih. Ergebn. Limnol., 21 : 341-350.

Lazzaro X., Drenner R.W., Stein R.A. \& Durward-Smith J. 1992. Planktivores and plankton dynamics : effects of fish biomass and planktivore type. Can. J. Fish. Aquat. Sci., 49 : 1466-1473.

Le Cohu R., Comoy N., Guitard J. \& Brabet J. 1991. — Périodicité du phytoplancton dans un réservoir de moyenne profondeur : le lac de Pareloup (Massif Central, France), un exemple de succession cyclique. Annls. Limnol., 27 (3) : 197-214.

Maier G. 1996. - Copepod communities in lakes of varying trophic degree. Arch. Hydrobiol., $136:$ 455-465.

McKee M.J. \& Knowles C.O. 1987. - Levels of protein, RNA, DNA, glycogen and lipid during growth and development of Daphnia magna Straus (Crustacea : Cladocera). Freshwat. Biol., $18: 341-351$.

McQueen D.J. \& Post J.R. 1988. - Limnocorral studies of cascading trophic interactions. Verh. Internat. Verein. Limnol., 23 (2) : 739-747.

McQueen D.J., Post J.R. \& Mills E.L. 1986. — Trophic relationships in freshwater pelagic ecosystems. Can. J. Fish. Aquat. Sci., 43 : 1571-1581.

McQueen D.J., Johannes M.R.S., Post J.R., Stewart T.J. \& Lean D.R.S. 1989. - Bottom-up and Top-down impacts on freshwater pelagic community structure. Ecol. Monogr., 53 (3) : 289-309.

McQueen D.J., Mills E.L., Forney J.L., Johannes M.R.S. \& Post J.R. 1992. - Trophic level relationships in pelagic food webs : comparisons derived from long-term data sets for Oneida Lake, New york (USA), and Lake St. George, Ontario (Canada). Can. J. Fish. Aquat. Sci., 49 : 1588-1596.

Meijer M.L., van Nes E.H., Lammens E.H.R.R., Gulati R.D., Grimm M.P., Backx J., Hollobeek P., Blaauw E.M. \& Breukelaar A.W. 1994. - The consequences of a drastic fish stock reduction in the large and shallow Lake Wolderwijd, The Netherlands. Can we understand what happened ? Hydrobiologia, 275/276:31-42.

Muck P. \& Lampert W. 1980. — Feeding of freshwater filter-feeders at very low concentration: poor evidence for «threshold feeding» and «optimal foraging» in Daphnia longispina and Eudiaptomus gracilis. J. Plankton Res., 2 :367-379.

Pourriot R. \& Leborgne L. 1970. — Teneurs en protéines, lipides et glucides du zooplancton d'eau douce. Ann. Hydrobiol., 1 (2) : 171-178.

Rey J. 1988. - Impact de la vidange sur le développement des populations zooplanctoniques dans une retenue des Pyrénées. Annls. Limnol., 24 (3) : 235-241.

Reyes-Marchand P. 1993. - Impact des alevins de gardons (Rutilus rutilus, L.) sur les communautés planctoniques littorales. Modélisation du fonctionnement trophique du lac d'Aydat. Thèse de Doctorat, Université Blaise Pascal, Laboratoire de Zoologie, Clermont II : $168 \mathrm{pp}$.

Richeux C., Arias-Gonzales J.E. \& Tourenq J.N. 1992. — Etude du régime alimentaire des gardons (Rutilus rutilus, L.) du lac de $\mathrm{Pa}$ reloup (Massif Central, France). Annls. Limnol., 28 (3) : 245-252.

Richeux C., Nogues J.F., Tourenq J.N. \& Aragon B. 1994a. - Inventaire piscicole de la retenue hydroélectrique de Pareloup lors de la vidange de juin 1993. Essai d'un nouveau système d'acquisition et de traitement des signaux d'un échosondeur. Hydroécol. Appl., 6 (1/2) : 197-226.

Richeux C., Dubois C., Arias-Gonzales J.E. \& Tourenq J.N. 1994b. - Régime et comportement alimentaire du gardon (Rutilus rutilus, L.) et de la perche (Perca fluviatilis, L.) de la retenue de Pareloup. II. Les adultes. Hydroécol. Appl., 6 (1/2) : 243-256.

Rudstam L.G., Lathrop R.C. \& Carpenter S.R. 1993. - The rise and fall of a dominant planktivore : direct and indirect effects on zooplankton. Ecology, 74 (2) : 303-319. 
Santer B. 1993. - Do cyclopoid copepods control Daphnia population in early spring, thereby protecting their juvenile instar stages from food limitation? Verh. Internat. Verein. Limnol., 25 : 634637.

Santer B. \& Lampert W. 1995. - Summer diapause in cyclopoid copepods : adaptative response to a food bottleneck ? J. Anim. Ecol., 64 (5) : 600-613.

Schindler J.E. 1971. - Food quality and zooplankton nutrition. $J$. Animal Ecology, 40 : 589-595.

Seda J. \& Duncan A. 1994. - Low fish predation pressure in London reservoirs : II. Consequences to zooplankton community structure. Hydrobiologia, 291 : 179-191.

Shapiro J. \& Wright D.I. 1984. — Lake restoration by biomanipulation : Round Lake, Minnesota, The first two years. Freshwat. Biol., 14 : 371-383.

Sicko-Goad L. \& Andresen N.A. 1991. - effect of growth and light/dark cycles on Diatom lipid content and composition. $J$. Phycol., 27 : 710-718.

Sprules W.G. 1984. - Towards an optimal classification of zooplankton for lake ecosystem studies. Verh. Internat. Verein. Limnol., $22: 320-325$.

Tedesco M.A. \& Duerr E.O. 1989. - Light, temperature and nitrogen starvation effects on the total lipid and fatty acid content and composition of Spirulina platensis UTEX 1928. J. Appl. Phycol., $1: 201-209$
Tessier A.J. \& Goulden C.E. 1982. - Estimating food limitation in cladoceran population. Limno. Oceanogr. 27 (4) : 707-717.

Townsend C.R. \& Perrow M.R. 1989. - Eutrophication may produce population cycles in roach (Rutilus rutilus, L.), by two contrasting mechanisms. J. Fish. Biol. 34 : 161-164.

Vanni M.J. 1986. - Fish predation and zooplankton demography : indirect effects. Ecology, 67 (2) : 337-354

Vanni M.J., Luecke C., Kitchell J.F. \& Magnuson J.J. 1990. — Effetcs of planktivorous fish mass mortality on the plankton community of lake Mendota, Wisconsin : implications for biomanipulation. Hydrobiologia, 200/201 : 329-336.

Vijverberg J. \& Frank TH. H. 1976. - the chemical composition and energy contents of copepods and cladocerans in relation to their size. Freshwat. Biol., $6: 333-345$.

Visman V., McQueen D.J. \& Demers E. 1994. - Zooplankton spatial patterns in two lakes with contrasting fish community structure. Hydrobiologia, 284 : 177-191.

Wilkinson L. 1989. - Systat : the system for statistics. Evanston, Illinois : systat INC. : $638 \mathrm{p}$

Yurkowski M. \& Tabachek J.L. 1979. - Proximate and amino acid composition of some natural fish foods. In : «Finfish nutrition and fishfeed technology». Halver \& Tiews Eds., Berlin, Vol. I : 435448. 


\section{Analyses d'ouvrages}

Adams D., Seitzinger S. \& Crill P. (Eds.) 1996. Cycling of reduced gases in the hydrosphere. Association Internationale de Limnologie Théorique et Appliquée. Communication $\mathrm{N}^{\circ}$ 25. 204 p. 92 fig., 45 tabl. E. Schweizerbart'sche Verlagsbuchhandlung (Nägele u. Obermiller) Stuttgart. ISBN 3-510-52025-4. DM 78 - US \$ 53.

Ce volume de 204 pages contient une partie des présentations effeectuées lors du. Symposium intitulé "Cycling of reduced gases in the Hydrosphere» organisé dans le cadre du $24^{\text {eme }}$ Congrès de l'Association Internationale de Limnologie Théorique et Appliquée (SIL), tenu à Munich (Allemagne) du 13 au 19 Août 1989: L'essentiel des articles originaux portant sur les cycles du méthane et de l'hydrogène dans l'eau, ils ont été complétés par des articles supplémentaires traitant de l'azote et du soufre, l'ensemble étant remis à jour (ou presque) jusqu'à la date de publication. L'intérêt particulier de ces gaz réduits ou partiellement oxydés (N2O) réside dans leurs rôles dans l'effet de serre, le cycle de l'ozone et les pluies acides.

Neuf articles sont consacrés à une revue générale des processus, trois aux dérivés gazeux de l'azote, trois aux dérivés gazeux réduits du soufre et cinq au méthane.

En introduction, D.D. Adams présente une revue des processus mis en oeuvre dans les cycles aquatiques et le transport des gaz réduits de l'hydrosphère vers l'atmosphère (article 1).

Deux articles sont consacrés à la méthodologie : Whiticar (article ${ }^{\circ} 4$ ) montre l'intérêt du traçage isotopique naturel $\left({ }^{13} \mathrm{C}\right)$ qui permet de différencier les processus et les réservoirs à l'origine du cycle du méthane. Dahm et al. (article $n^{\circ} 9$ ) utilisent la spectroscopie infra-rouge (F.T.I.R.) pour mesurer in situ les concentrations en $\mathrm{H} 2 \mathrm{O}, \mathrm{CH} 4, \mathrm{~N} 2 \mathrm{O}$ et $\mathrm{CO}$ de l'air sur un long trajet (10 à $1000 \mathrm{~m}$ ) au-dessus des systèmes aquatiques. Le couplage des concentrations avec les méthodes micro-météorologiques devrait permettre les évaluations des flux.

Comme le montre Conrad (article $n^{\circ} 2$ ), l'hydrogène est au centre de la décomposition anaérobie et joue le rôle de régulateur. Cependant, ce gaz est maintenu à de faibles concentrations par des microorganismes oxydants et a peu d'influence sur le cycle atmosphérique (Kuivila \& Lovley, article $n^{\circ} 5$ ).

La compétition entre les sulfato-réducteurs et les méthanogènes est décrite par Zaiss (article $n^{\circ} 6$ ) dans différents milieux et par Westermann et Ahring (article $n^{\circ} 7$ ) dans une aulnaie. En présence de sulfate, la compétition est en faveur des sulfato-réducteur et la méthanogénèse se fait à partir de la réduction du $\mathrm{CO}_{2}$. Dans les milieux d'eau douce, la méthanogénèse domine mais à partir de l'acétate.

Les émissions de méthane et les facteurs régulateurs ont fait l'objet de nombreuses études. L'influence des facteurs climatiques à l'échelle planétaire est mise en évidence par Crill (article $\mathrm{n}^{\circ} 16$ ). Les variations locales, spatiales et saisonnières sont illustrées dans la plaine amazonienne par Devol et al. (article $n^{\circ} 17$ ) et Wassman \& Thein (article $n^{\circ} 18$ ) ou dans les rivières de la côte pacifique U.S. par Liley et al. (article ${ }^{\circ} 19$ ).

L'absence d'émission peut être due à l'absence de certains microorganismes du consortium méthanogène (Naguib \& Adams, article $n^{\circ} 8$ ). Plus généralement, les bactéries méthanotrophes consomment le méthane 
à la surface des sédiments (King \& Blackburn, article $\mathrm{n}^{\circ} 3$, Sweerts et al., article $\mathrm{n}^{\circ} 20$ ).

L'influence du protoxyde d'azote $\mathrm{N}_{2} \mathrm{O}$ sur l'ozone stratosphérique est une motivation forte de l'étude des émissions. Capone \& Costanza (article $n^{\circ} 11$ ) travaillent, à l'échelle des océans, par l'étude des bilans d'azote. Wada et al. (article $n^{\circ} 12$ ) font la part de la nitrification et de la dénitrification comme source de $\mathrm{N}_{2} \mathrm{O}$ par traçage isotopique naturel $\left(\delta^{15} \mathrm{~N}\right)$. Terai et Yoh (article $n^{\circ} 10$ ) mesurent la dénitrification dans la colonne d'eau et les sédiments du lac Zizaki au Japon. L'activité maximale est observée fin juin, juillet et la zone littorale est, en moyenne, la plus productive.

Les gaz sulfureux jouent un rôle important dans la chimie atmosphérique mais aussi dans le bilan énergétique (absorption infra-rouge, aérosols). Hines (article $n^{\circ} 15$ ) fait le bilan des connaissances sur les émissions de gaz sulfureux par les zones humides. Les données concernant les zones humides continentales sont peu nombreuses et doivent être complétées pour améliorer les connaissances du cycle.

Le diméthyl sulfure (D.M.S.) est le composé volatile principal émis par les zones humides salées ou d'eau douce. Son origine est liée aux algues et aux plantes supérieures. Le D.M.S. constitue $90 \%$ des composés réduits du soufre émis dans l'atmosphère (Kiene, article $\mathrm{n}^{\circ} 13$ ).

Comme on le voit, cet ouvrage permet de se faire une idée des connaissances actuelles sur les émissions de composés gazeux réduits qui interfèrent avec les équilibres atmosphériques. Les articles sont accompagnés d'une bibliographie abondante, peut-être un peu ancienne du fait du retard dans l'édition.

Ce volume intéressera tous les chercheurs, biologistes ou physiciens qui travaillent sur ces sujets, mais aussi tous ceux qui veulent se tenir informés des phénomènes qui gravitent autour du fameux «global change» et de l'avenir incertain de la planète.

\section{Labroue}

Cook (C.D.K.) 1996. - Aquatic Plant Book, SPB Academic Publishing, Amsterdam : 228 p. ISBN 90-5103-132-7. Dfl 110.00, US \$ 69.00.

Cet ouvrage est un guide des plantes vasculaires aquatiques du monde. Il s'agit d'une seconde édition révisée de l'ouvrage publié en 1990 et qui succédait au livre de Cook et al. (1974) intitulé «Water plants of the World». Cette nouvelle édition prend en compte les dernières révisions de la nomenclature internationale et présente 407 genres répartis dans 87 familles différentes, appartenant aux Ptéridophytes et aux Sper- matophytes. Les genres considérés sont ceux qui incluent des espèces que l'on peut trouver dans l'eau : totalement submergées, vivant les pieds dans l'eau, ou bien encore flottant à la surface et qui colonisent aussi bien des systèmes permanents ou semi-permanents d'eau douce que d'eau salée (zones tidales comprises) partout dans le monde.

Après avoir défini le vocabulaire de base utilisé, l'auteur présente 10 clés de détermination, la première étant établie sur les caractères végétatifs et permettant de différentier les groupes principaux. L'utilisateur est ainsi aiguillé vers la ou les clés les plus appropriées pour la détermination d'une famille ou d'un genre donné sans pour cela avoir recours systématiquement à un microscope.

Pour chaque genre, l'auteur indique une estimation du nombre total d'espèces connues et une estimation du nombre d'espèces aquatiques, ainsi que là répartition naturelle de ce genre et les zones ou certaines espèces ont été introduites. Il décrit ensuite classiquement les caractères morphologiques du genre, mais donne également des informations sur son écologie, les formes de vie pour les stades adultes et ce qui est moins courant pour les stades juvéniles, ainsi que sur les mécanismes de pollinisation, sur les diaspores et leur(s) mode(s) de dissémination, sur l'utilisation de certaines espèces et leur intérêt économique. Enfin, l'auteur renvoie le lecteur à des références plus complètes et relativement récentes concernant le genre présenté. On peut regretter que dans cet ouvrage chaque genre ne soit illustré que par une seule espèce aquatique. Toutefois, 408 figures (réparties en 130 planches en noir et blanc) simples et didactiques illustrent les propos de l'auteur et permettent d'identifier les principales espèces aquatiques. Pour certaines d'entre elles, des dessins de détails permettent de visualiser le ou les caractères distinctifs et facilitent ainsi leur identification.

Cet ouvrage est donc remarquable à plus d'un titre et peut apporter des informations utiles non seulement aux botanistes mais à toutes les personnes qui s'intéressent de près ou de loin aux milieux aquatiques. On regrettera seulement le format utilisé qui ne permet pas de transporter facilement cet ouvrage sur le terrain.

A.M. Planty-Tabacchi

Arrignon J. 1996. — L'écrevisse et son élevage. $3^{\mathrm{e}}$ édition. Lavoisier, Technique et Documentation, Paris : 230 p. ISBN 2.7430.0064.3. 250F.

Il s'agit de la $3^{\mathrm{e}}$ édition revue et augmentée de cet ouvrage entièrement consacré aux écrevisses qui peu- 
plent actuellement les eaux douces d'Europe occidentale, qu'elles soient indigènes ou qu'elles aient été introduites.

Outre une courte introduction qui retrace la paléogéographie de ces Décapodes dulçaquicoles et l'origine des peuplements européens, cet ouvrage comporte cinq chapitres. Les deux premiers pourraient être regroupés en une première partie car ils apportent les connaissances de base sur ce crustacé «géant» de nos eaux douces. Ils présentent une description très poussée de la morphologie générale des Astacidés, de leur anatomie et des principales fonctions de relations (organes des sens, appareils circulatoire, respiratoire, excréteur, génital ...). Cette présentation générale des Astacidés se prolonge logiquement par une clé d'identification des 7 espèces et des sous espèces d'écrevisses que l'on peut rencontrer en Europe de l'ouest. Cette clé, d'utilisation facile, est bien illustrée à l'aide de schémas très explicites et de photos. Cette partie se prolonge par une présentation de la distribution des diverses espèces en France (illustrée par des cartes découpées selon les limites des départements) mais aussi dans certains pays voisins tels la Suisse et la Belgique ou dans diverses régions du monde dont l'Australie. Dommage que la qualité d'impression des cartes de distribution ne soit pas excellente ce qui rend parfois difficile la lecture des indications qu'elles portent.

Le chapitre suivant, entièrement consacré à la biologie et à l'écologie de ces Décapodes, pourrait constituer la $2^{\text {enc }}$ grande partie de cette monographie. Il peut être découpé en trois sections dont la première traite de la biologie générale des représentants de la famille des Astacidés et notamment de toute la phase de reproduction : de l'accouplement à l'éclosion en passant par la ponte et l'incubation. Sont également décrits en détail tous les problèmes relatifs au développement, à la mue en relation avec le métabolisme calcique, à la régénération des appendices après amputation, à la croissance et à l'alimentation. L'auteur combat certaines idées reçues et notamment celle qui présente les écrevisses comme des nécrophages : bien qu'à l'occasion elles puissent se nourrir de cadavres, ce sont les organismes vivants qui constituent la part dominante de leur alimentation. La nature des proies et des aliments ingérés par une espèce d'écrevisse en fonction de l'âge montre bien l'importance des invertébrés dans le bol alimentaire.

Une synthèse des divers aspects du comportement clôt cette analyse générale de la biologie et de l'écologie des Astacidés.
Ces données seront reprises dans la $2^{\mathrm{eme}}$ section qui est une étude espèce par espèce : l'auteur a établi pour chacune des espèces susceptibles d'être observées en Europe une fiche comparative où sont regroupées les caractéristiques biologiques et écologiques; trois formes australiennes (genre Cherax) dont l'introduction en région paléarctique a échoué mais dont la réintroduction est toujours possible par le biais de l'aquariophilie, sont également présentées.

La $3^{\text {eme }}$ section est consacrée à l'écologie des populations; celle-ci est étudiée, comme toujours, à partir d'enquêtes et d'inventaires souvent réalisés par des méthodes de capture/recapture avec ou sans marquage des individus. La dynamique des populations d'écrevisse en milieu naturel est illustrée par des exemples très démonstratifs issus de la littérature. Les facteurs limitants des populations sont analysés ensuite. Il apparaît que deux sont particulièrement importants : la pollution et les maladies. L'altération de la qualité de l'eau et surtout la destruction directe (barrages, remembrement ou modification de la géométrie des cours d'eau, recalibrage des berges) des habitats astacicoles sont responsables du déséquilibre constaté depuis une trentaine d'années dans les populations d'écrevisses. Les maladies, d'origines multiples, sont présentées en détail dans cet ouvrage; seules des mesures prophylactiques semblent pouvoir réduire cette cause de limitation, voire d'anéantissement, des populations. Ces maladies sont par ailleurs favorisées par les pollutions chimiques qui affaiblissent les individus et les rendent plus sensibles aux épidémies.

Parmi les maladies parasitaires l'auteur signale «de petites sangsues du genre Branchiodella». Notons que les Branchiodellidae ont été alternativement classés parmi les Oligochètes ou les Hiridinées (sangsues) mais qu'ils sont actuellement considérés comme un groupe distinct parmi les Annélides.

La prédation (malgré la longue liste de prédateurs établie par l'auteur à partir des données de la littérature) ne fait l'objet que d'un court paragraphe tant son action dans la limitation des populations est réduite par rapport à celle des pollutions et des maladies.

La fin de ce long chapitre clôt la partie fondamentale de cet ouvrage qui fait la synthèse de toutes les connaissances acquises sur la taxonomie, la biologie et l'écologie au cours de nombreuses recherches en milieu naturel sur diverses espèces d'écrevisses.

De par leur intérêt pour la pêche et dans l'alimentation humaine, les écrevisses ont également donné lieu à une recherche appliquée dont les principaux résultats sont récapitulés dans le chapitre suivant consacré à l'élevage. Il constitue un véritable guide pratique qui 
passe en revue tous les problèmes relatifs à la réalisation d'un élevage : choix des espèces, objectifs (repeuplement ou consommation), divers types de milieux (plans d'eau, étangs, canaux, fossés, bassins artificiels), écloseries ainsi que les divers dispositifs et accessoires nécessaires. Des plans de bâtiments sont même proposés.

Les problèmes spécifiques aux divers types d'élevages sont ensuite analysés : méthodes de fertilisation du milieu, d'alimentation et de récolte pour les élevages extensifs, incubation, nourrissage artificiel, stockage et transport pour les élevages intensifs. Cette partie se termine par une analyse de la rentabilité des élevages à l'aide d'exemples de bilans financiers coûts - revenus.

La dernière partie de cet ouvrage, qui correspond au dernier chapitre n'est plus du domaine scientifique; elle traite exclusivement de problèmes économiques liés soit à la consommation des écrevisses soit à la pêche de loisir.

Concernant la consommation, cet ouvrage présente une étude de marché dans les divers grands pays producteurs et/ou consommateurs d'écrevisses dans le monde avec une large place réservée au marché français. Sont également abordés les problèmes d'évolution du marché avec ses aléas et ses perspectives.

L'aspect économique lié à la pêche de loisir débute par un inventaire des divers modes de pêche etse termine avec les problèmes relatifs à la gestion des peuplements et notamment la règlementation en vigueur relative à cette pratique de pêche (une compilation des textes règlementaires est fournie). La gestion des peuplements implique, outre une règlementation, la mise au point de stratégies d'aménagement.

L'auteur met en garde contre certaines méthodes de gestion qui se sont souvent soldées par des échecs comme par exemple celles qui sont basées uniquement sur le concept de rendement maximum supportable (RMS); il plaide en faveur d'une gestion basée sur la connaissance des peuplements. Celle-ci suppose des moyens financiers permettant d'analyser la situation de chacun des peuplements afin d'élaborer une stratégie d'aménagement rationnelle en fonction du but recherché : le loisir ou la consommation.

Cet ouvrage se veut être un essai de synthèse des connaissances les plus utiles et les plus exploitables par le naturaliste, le pêcheur et l'économiste. Il a pleinement atteint son objectif. L'écrevisse étant souvent utilisée en enseignement comme type de crustacé, cet ouvrage est aussi à conseiller à tous les enseignants de biologie animale qui ont à présenter cet animal à leurs étudiants.

\section{N. Giani}

Petts G. \& Calow P. (Eds.). 1996. - River Biota. Diversity and dynamics. Blackwell Science Ltd. 257 p.; ISBN 0.86542-716-X, £ 24.95.

Cet ouvrage qui est un condensé des deux volumes antérieurs : The Rivers Handbook $(1992,1994)$ décrit les organismes vivants dans les rivières, les taxons, adaptation, écologie et relations trophiques. Cinq chapitres sont consacrés aux organismes, selon une hiérarchie trophique allant des algues aux poissons; en passant par les décomposeurs microbiens et les invertébrés. Ceci conduit à l'étude des interactions et des réseaux trophiques (chap.7) et aux processus associés à la photosynthèse et à la décomposition. Le chapitre 10 traite des principes généraux liés à l'échantillonnage et aux traitements des données; de nombreuses références bibliographiques y sont indiquées. Les deux derniers chapitres $(11,12)$ s'attachent à quelques exemples d'impacts d'activité humaines sur la rivière et montrent comment les biocénoses peuvent être utilisées en tant qu'indicateurs pour en estimer les effets.

Cet ouvrage d'un format et d'une présentation trés agréables, traite des principaux sujets associés à l'étude des rivières. Si certains processus ne sont pas abordés, celà est généralement compensé par une trés abondante bibliographie. D'un format et d'un prix plus réduits que «The Rivers Handbook», cet ouvrage les remplace avantageusement : il est à recommander d'abord aux étudiants qui y trouveront de quoi largement approfondir leurs connaissances sur les processus en rivière mais aussi aux chercheurs qui apprécieront son style et l'approche synthétique des phénomènes étudiés au travers d'une bibliographie assez exhaustive.

A. Dauta 


\section{(C) 1997}

Tous droits de traduction, d'adaptation et de reproduction par tous procédés réservés pour tous pays.

La loi du 11 mars 1957, n'autorisant aux termes des alinéas 2 et 3 de l'article 41 , d'une part, que les copies ou reproductions strictement réservées à l'usage privé du copiste et non destinées à une utilisation collective et, d'autre part, que les analyses et courtes citations dans un but d'exemple et d'illustration, " toute représentation ou reproduction intégrale, ou partielle, faite sans le consentement de l'auteur ou de ses ayants droit ou ayants cause, est illicite " (alinéa ler de l'article 40).

Cette représentation ou reproduction, par quelque procédé que ce soit, constituerait donc une contrefaçon sanctionnée par les articles 425 et suivants du Code pénal.

Dépôt légal : $2^{\mathfrak{c}}$ trimestre 1997

Imprimerie du Champ-de-Mars - 09700 SAVERDUN

Printed in France. 